\title{
Surgical Management of Endometriosis- A Mini Review
}

\section{Kanika Chopra*, Debasis Dutta and Kanika Jain}

Department of Minimally Invasive Gynaecology, Institute of Obstetrics and Gynaecology, Sir Ganga Ram Hospital, India

"Corresponding author: Chopra K, Department of Minimally Invasive Gynaecology, Institute of Obstetrics and Gynaecology, Sir Ganga Ram Hospital, New Delhi, India, Tel: 09958121123; E-mail: kank2kanu@ yahoo.co.in

Received: October 14, 2018; Accepted: October 27, 2018; Published: October 30, 2018

\begin{abstract}
Endometriosis is the most devitalizing disease affecting the reproductive age women and is usually characterized by 4 D's i.e. dysfunctional uterine bleeding, dysmenorrhea, dyspareunia and dyschezia. Now days, the detection of the disease and its management is simplified by the presence of 4D ultrasounds, MRI and laparoscopy. In majority patients, surgical management by minimally invasive route is the answer and so, the aim of this review is to highlight the issue in brief.
\end{abstract}

Keywords: Endometriosis; Menstruation; Endometrial cells

\section{Introduction}

Endometriosis is a chronic debilitating disease affecting around 6-10\% of reproductive age women [1]. Various theories have been postulated detailing the development of endometriosis. These are metaplasia of coelomic epithelium, benign metastasis via blood vessels and lymphatics spread of endometrial cells, retrograde menstruation as proposed by Sampson [2-4]. The anatomic distribution of endometriotic lesions favour retrograde menstruation theory, with superficial implants most commonly located in posterior compartment of the pelvis and in left hemipelvis $[5,6]$. There exists high evidence of immune system dysregulation as one of the causes of implantation and growth of endometrial cells in ectopic locations and is still under extensive research.

Endometriosis is related to chronic pelvis pain in majority affecting their quality of life and also infertility. It may even be asymptomatic in majority. The gold standard for making a definitive diagnosis is by laparotomy or laparoscopically and histopathological examination of the lesions identified. The different forms of endometriotic lesions are superficial peritoneal implants, endometriomas and deep infiltrative endometriosis involving rectovaginal septum. Peritoneal implants are most commonly seen in the uterosacral ligaments, pouch of douglas, ovarian fossae and pelvic side walls. Less frequently, bladder and bowel are involved and rarely upper abdomen as well. Gastrointestinal involvement is seen is 3\%-37\% cases, with severe endometriosis affecting uterosacral ligaments, rectovaginal septum, rectosigmoid colon and appendix [7,8]. Genitourinary endometriosis is seen in $1 \%-2 \%$ cases with ureter involvement in $0.1 \%-0.2 \%$ cases, with majority affecting distal ureter [9- 
www.yumedtext.com | October-2018

11]. Typically, the left ureter is involved and endometriosis leads to extrinsic compression of the ureter leading to irreversible damage to renal function. This, thus warrants timely diagnosis and management.

The nature and the appearance of the lesions also vary to a very large extent. Powder burnt lesions represent foci of inactive disease and usually are asymptomatic [12]. Atypical or non-pigmented lesions on the other hand are seen as clear vesicles, white scarred lesions, red lesions, yellow brown patches and peritoneal windows. These lesions are active endometriotic lesions and secrete prostaglandins [13]. Ovaries are usually the most common pelvic organs involved in this debilitating disease leading to formation of endometriomas. Deep infiltrating endometriotic lesions are usually retroperitoneal with minimal peritoneal involvement.

ASRM, American Society for Reproductive Medicine, 1996 has been widely accepted to stage the disease and it includes four groups, stage 1(minimal), stage 2(mild), stage 3(moderate) and stage 4 (severe) [14,15]. The type of treatment offered to the patient, whether medical or surgical depends to a larger extent on the age of the patient, desire of the patient, especially if the main complaint is infertility, extent and the severity of the disease. Here, my main aim is to discuss about the surgical management of endometriosis laparoscopically.

Nowadays, minimally invasive technique with enhanced vision and lightning is an answer to all those patients suspected of endometriosis as it helps in definite diagnosis and treatment simultaneously. It can be conservative or radical as explained below very efficiently in the hands of experienced surgeon with maximum patient benefit in the form of minimal postoperative pain and early recovery.

\section{Conservative Surgery}

Women desirous of pregnancy and in those the disease leads to pain involving vital organs like bladder and bowel, conservative surgical approach is treatment of choice. This involves removal of all visible implants and adhesiolysis with the main aim of restoring the tubo- ovarian relationship. There definitely exists high risk of recurrence of the disease and thus the need for repeat surgery for the same in $25 \%$ cases [16]. In patients with minimal or mild endometriosis associated with infertility, a metanalysis of studies showed that either no treatment or surgery was found to be superior to medical treatment for its management [17]. Both fecundity and pain relief are definitely improved with surgery in patients with advanced stage disease [18].

Superficial peritoneal endometriotic implants less that $2 \mathrm{~mm}$ are vaporised with laser, coagulated with monopolar or bipolar current or excise and in those more than $3 \mathrm{~mm}$ excision is required. Ovarian endometriomas are best managed by laparoscopic ovarian cystectomy via stripping technique.

\section{Radical Operation}

Patients not desiring future fertility and suffering severely from this debilitating disease or patients who fail to respond to conservative management are candidates for radical ablative procedure. Even patients with severe endometriosis involving vital structures like bowel, bladder, ureter may require radical surgery for the appropriate management of the same. This 
www.yumedtext.com | October-2018

involves oophorectomy, salpingo-opherectomy, hysterectomy, excision of deep infiltrating endometriosis. In cases of involvement of bowel, resection may be required for complete cure. Similarly, if less than $2 \mathrm{~cm}$ length of the ureter is involved, resection is done followed by end to end anastomosis. In cases of involvement of longer lengths, ureteric implantation may be the answer. In cases of bladder endometriomas, partial cystectomy with primary repair of cystotomy is the treatment of choice if adequate post resection capacity is being achieved.

\section{Recurrence after Surgery}

The chances of recurrence of endometriosis varies greatly from $2 \%-47 \%$, highest percentage seen for rectocervical disease. In patients, in whom conservative procedure is done and who desire fertility, the best treatment is patient achieving pregnancy. In other cases, aromatase inhibitors like letrozole and progestin norethindrone acetate along with calcium and vitamin D supplements for at least six months helps in decreasing pain score considerably [19]. In cases, where radical surgery was done, but still foci of endometriotic lesions have been left behind owing to its close proximity to vital structures, low dose hormone replacement therapy is given with tibolone supposed to be one of the safest option available.

\section{Recent Advances}

Among the various medical treatment options available, the main mechanism behind them is creating hypo estrogenic environment, which thus have its own limitations and side effects. Addressing these issues, the various treatment options under study are, aromatase inhibitors, selective estrogen receptor modulators and selective progesterone inhibitors and are under investigation. These are aimed to specifically target endometrial implants. ${ }^{19}$

Our experience in dealing with endometriosis surgically is vast, from ovarian endometrioma to bowel endometriosis involving rectosigmoid, bilateral uterosacral ligaments completely obliterating the pouch of douglas as can be seen in FIG. 1 and 2; and also bladder endometriosis. We have realised over the years, that laparoscopy with its advanced magnification and lighting helps the surgeons in providing the best treatment and relief for the patient.

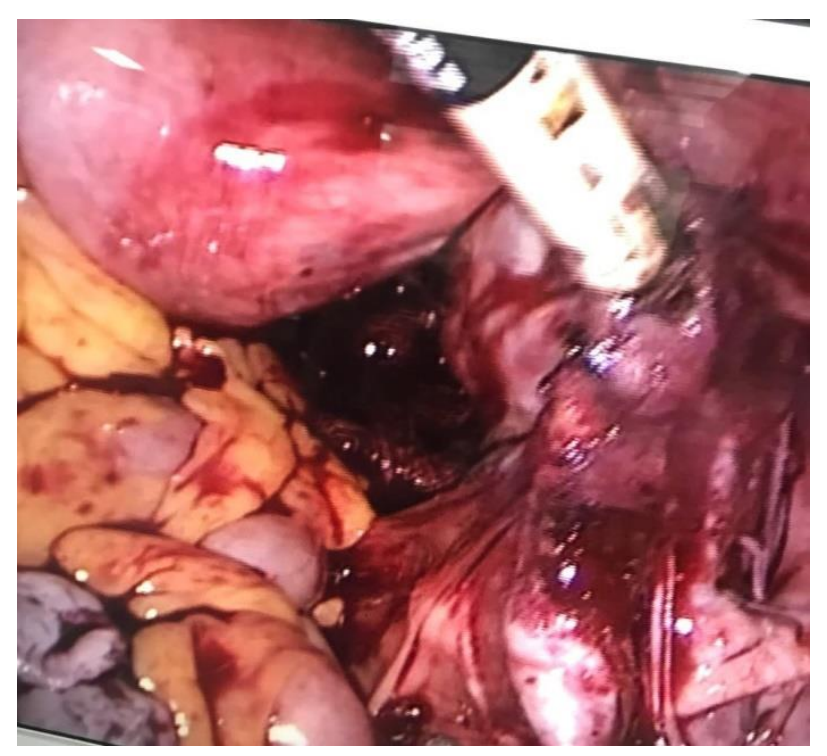

Fig. 1 


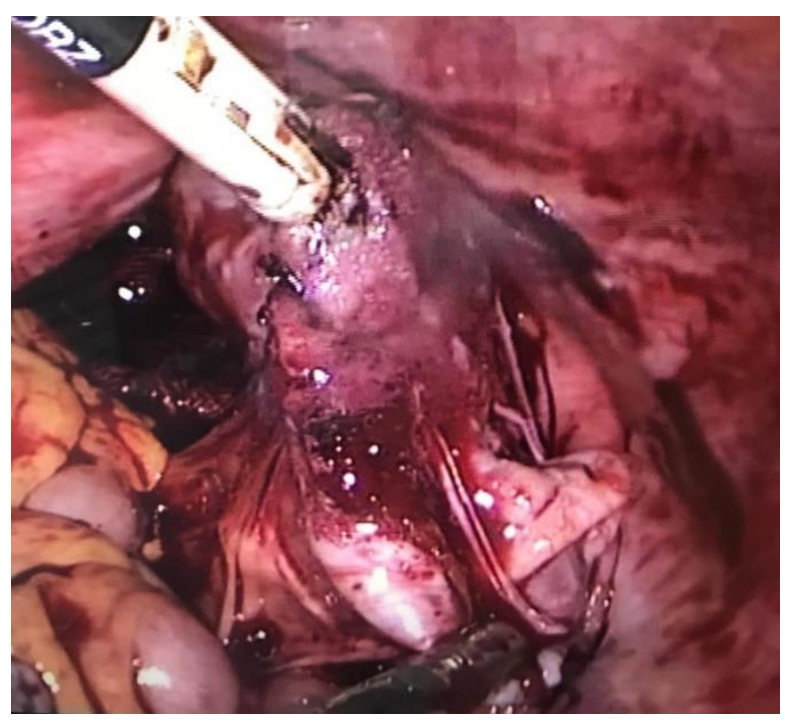

Fig. 2

FIG. 1 and 2. Rectosigmoid being separated from endometrioma.

\section{Conclusion}

The patients diagnosed with endometriosis usually suffer from chronic pelvic pain and infertility and thus warrants surgical management and laparoscopy offer the best route for the same. All gynaecologists dealing with endometriosis should be well aware of the need and the extent of surgery required for the appropriate management of the patient to give optimal results.

\section{REFERENCES}

1. Eskenazi B, Warner ML. Epidemiology of endometriosis. Obstet Gyncol Clin North Am. 1997;24:235-58.

2. Iwanoff NS. Adenofibroma cysticum sarcomatodes carcinomatosum. Monatsch Geburtshilfe Gnakol.1898;7:295300.

3. Halban J. Metastatic hysteroadenosis.Wein Klin Wochenschr.1924;37:1205-6.

4. Sampson JA. Perforating hemorrhagic cysts of the ovary;their importance and especially their relation to pelvic adenomas of endometrial type( adenomyoma of the uterus, rectovaginal septum, sigmoid, etc). Arch Surg. 1921;3(2):245-323.

5. Dmowski WP, Radwanska E. Current concepts on pathology, histogenesis and etiology of endometriosis. Acta Obstet Gynecol Scand. 1984;123:29-33.

6. Al- Fozan H, Tulandi T. Left lateral predisposition of endometriosis and endometrioma. Obstet Gynecol. 2003;101(1):164-6.

7. Jenkinson EL, Brown WH. Endometriosis: a study of 117 cases with special reference to constricting lesions of the rectum and sigmoid colon. JAMA. 1943;122:349.

8. Samper ER, Sagle GW, Hand AM. Colonic endometriosis, its clinical spectrum. South Med J. 1984;77:912.

9. Nezhat C, Hood J, Winer W, et al. Videolaseroscopy and lasee laparoscopy in gynaecology. Br J Hosp Med. 1987;38(3): 219-24.

10. Antonelli A, Simeone C, Frego E et al. Surgical treatment of ureteral obstruction from endomtriosis: our experience with thirteen cases. Int Urogynecol J Pelvic Floor Dysfuct. 2004;15(6):407-12. 
www.yumedtext.com | October-2018

11. Zanetta G, Webb MJ, Segura JW. Ureteral endometriosis diagnosed at ureteroscopy. Obstet Gynecol. 1998;91(5 pt 2):857-9.

12. Martin DC, Hubert GD. Laparoscopic appearances of pelvic endometriosis. Fertil Steril. 1989;51(1):63-7.

13. Nisolle M, Donnez J. Peritoneal endometriosis, ovarian endometriosis, and adenomyotic nodules of the rectovaginal septum are three different entities. Fertil Steril. 1997;68(4):585-96.

14. American Society for Reproductive Medicine. Revised American Society for Reproductive Medicine classification of endometriosis: 1996. Fertil Steril. 1997;67(5):817-21.

15. The American Fertility Society. Revised American Fertility Society classification of endometriosis: 1985 . Fertil Steril. $1985 ; 43(3): 351-2$.

16. Schenken SR, Malink RL. Reoperation after initial treatment of endometriosis with conservative surgery. Am J Obstet Gynecol. 1978;131(4):416-24.

17. Adamson GD, Pasta DJ. Surgical treatment of endometriosis associated infertility: meta- analysis compared with survival analysis. Am J Obstet Gynecol. 1994;171(6):1488.

18. Chaperon C, Fritel X, Dubisson JB. Fertility after laparoscopic management of deep endometriosis infiltrating the uterosacral ligaments. Hum Reprod. 1999;14(2):329-32.

19. Chwalisz K, Garg R, Brenner RM, et al. Selective progesterone receptor modulators (SPRMs): a novel therapeutic concepts in endometriosis. Ann N Y Acad Sci. 2002;955:373-88. 